\title{
Uso das intervenções de enfermagem na prática clínica no Brasil ${ }^{1}$
}

\author{
The nursing interventions used at clinical practice in Brazil
}

\section{La intervenciones de la enfermería utilizadas en la práctica clínica en lo Brazil}

\author{
Tânia Couto Machado Chianca', Cristiane Chaves de Souza"', Andreza Werli"',
} Fernanda Luiza Hamze ${ }^{I V}$, Flávia Falci Ercole ${ }^{V}$

\begin{abstract}
${ }^{1}$ Resultados da pesquisa “Aplicabilidade das Intervenções de Enfermagem da NIC no Brasil” financiada pelo CNPq e FAPEMIG.
' Enfermeira. Doutorada em Enfermagem. Professor associado da Escola de Enfermagem da Universidade Federal de Minas Gerais. E-mail: tchianca@enf.ufmg.br.

"Enfermeira. Mestranda em Enfermagem pela Escola de Enfermagem da Universidade Federal de Minas Gerais. E-mail: crismatipo@yahoo.com.br.

II'Enfermeira. Mestranda em Enfermagem pela Escola de Enfermagem da Universidade Federal de Minas Gerais. E-mail: andwe20@yahoo.com.

${ }^{I V}$ Enfermeira. Hospital Universitário Risoleta Tolentino Neves, Belo Horizonte/MG. E-mail: fernandahamze@hotmail.com.

$\checkmark$ Enfermeira. Doutorado em Parasitologia pela Universidade Federal de Minas Gerais. Professor adjunto da Universidade Federal de Minas Gerais. E-mail: fercole@enf.ufmg.br.
\end{abstract}

\section{RESUMO}

Há um desafio mundial de universalizar a linguagem e evidenciar a prática de enfermagem. A Nursing Interventions Classification ( NIC) está sendo desenvolvida por pesquisadores do College of Nursing - The University of Iowa, EUA, com esse propósito. Este estudo teve por objetivo identificar o uso das intervenções de enfermagem da NIC no Brasil, por meio de levantamento da freqüência de utilização das 486 intervenções da NIC nas diversas especialidades clínicas dos enfermeiros brasileiros. Trata-se de estudo descritivo, realizado no período de março a julho de 2006 , com amostra de 222 enfermeiros. Para a coleta de dados foi utilizado um instrumento aplicado pelo grupo de pesquisadores de lowa. Para a análise dos dados foi utilizado um pacote estatístico. Um grau de concordância de $70 \%$ foi considerado aceitável e as intervenções centrais, consideradas como utilizadas diariamente ou semanalmente pelos enfermeiros foram identificadas. Os resultados mostraram que $77,7 \%$ das intervenções $\mathrm{NIC}$ foram identificadas como essenciais por enfermeiros de diferentes especialidades. As intervenções mais freqüentes mostram a essência da prática clínica da enfermagem no Brasil e a aplicabilidade das intervenções NIC à nossa realidade.

Descritores: Enfermagem; Classificação; Cuidados de Enfermagem.

\section{ABSTRACT}

There is a challenge in the world to standardize the nursing language and to give evidence to the nursing practice. Nursing Interventions Classification (NIC) is being developed by researchers of the College of Nursing - The University of I owa, USA, with that purpose. The objective of this study was to identify the use of the NIC interventions in Brazil, through a survey of the 486 NIC interventions frequency of use by Brazilian nurses from several clinical specialties. A descriptive study carried out from March to July of 2006 with a sample of 222 Brazilian nurses was conducted. An instrument applied by the lowa group of researchers was used for data collection. A statistic package was used for the data analysis. An agreement of $70 \%$ was considered acceptable and the central interventions considered used daily or weekly by the nurses were identified. The results showed that $77,7 \%$ of the NIC interventions were identified as essential for the nurses of different specialties. The most frequent interventions show the essence of the nursing practice in Brazil and also the applicability of NIC interventions to our reality.

Descriptors: Nursing; Classification; Nursing care.

\section{RESUMEN}

Hay un desafío mundial para regularizar el idioma de la enfermería y dar evidencia a la práctica de la enfermería. La Clasificación de las Intervenciones de Enfermería (NIC) está siendo desarrollada por investigadores de la Facultad de Enfermería - La Universidad de Iowa, EE.UU., con ese propósito. El objetivo de este estudio fue identificar el uso de las intervenciones NIC en Brasil, a través de un estudio de la frecuencia de uso de las 486 intervenciones por las enfermeras brasileñas de varias especialidades clínicas. Un estudio descriptivo llevado a cabo de marzo a julio de 2006 con una muestra de 222 enfermeras brasileñas. Un instrumento aplicado por el grupo de investigadores de Iowa fue usado para la colección de datos. Un paquete estadístico se usó para el análisis de los datos. Un acuerdo de $70 \%$ fue considerado aceptable y las intervenciones centrales consideradas de usos periódicos o usados toda semana por las enfermeras fueron identificadas. Los resultados mostraron que $77,7 \%$ de las intervenciones NIC fueron identificadas como esenciales por las enfermeras de diferentes especialidades. Las intervenciones más frecuentes muestran el ser de la práctica de la enfermería en Brasil y también la pertinencia de las intervenciones NIC a nuestra realidad.

Descriptores: Enfermería; Clasificación; Atención de Enfermería. 


\section{NTRODUÇÃO}

A enfermagem tem evoluído ao romper com o estereótipo centrado nas técnicas durante a assistência e focalizado o indivíduo como um todo no cuidado prestado. Esta evolução foi facilitada pelas pesquisas, principalmente na área de teorias de enfermagem, que tem proporcionado mudanças no paradigma do conceito de saúde. Classificar a prática tem sido um dos mais significativos trabalhos desenvolvidos por estes profissionais no mundo(1).

Dentre os trabalhos mais significativos desenvolvidos pela enfermagem em todo o mundo encontra-se a classificação de elementos de sua prática, como os diagnósticos, intervenções e resultados de enfermagem. Existe um desafio mundial de universalizar a linguagem utilizada pelos enfermeiros e tornar a prática de enfermagem mais evidente. A utilização dos sistemas de classificação na prática de enfermagem tem mobilizado os enfermeiros em todo o mundo que buscam dar maior visibilidade à profissão. Os sistemas classificatórios contêm termos que designam o que a enfermagem identifica, trata e avalia em seus pacientes, e estes são elementos importantes na busca de cuidados mais qualificados aos pacientes justificando a necessidade de utilização e classificações para incrementar a prática clínica de enfermagem ${ }^{(1-2)}$.

Dentre os sistemas de classificação de linguagens de enfermagem disponíveis, encontra-se a Classificação das Intervenções de Enfermagem (Nursing Interventions Classification - NIC), desenvolvida desde 1987, por um grupo de pesquisadoras do "College of Nursing" - The University of Iowa, EUA, como parte de um projeto de estudos sobre as intervenções de enfermagem ${ }^{(1)}$. A NIC descreve as intervenções que a enfermagem executa e foi embasada em estudos e na prática clínica dos enfermeiros. As intervenções são definidas como "qualquer tratamento, baseado no julgamento e conhecimento clínico, realizado por um enfermeiro para aumentar os resultados do paciente/cliente"(1). Cada intervenção possui título, definição e uma lista de atividades a serem executadas. As intervenções estão agrupadas em classes que, por sua vez são referentes a sete domínios: fisiológico básico, fisiológico complexo, comportamental, segurança, família, sistema de saúde e comunidade ${ }^{(1)}$.

No Brasil, existem lacunas no conhecimento sobre a NIC. Estudos devem ser desenvolvidos para a identificação, comparação, utilização, validação e adequação de termos utilizados nesta classificação. Dessa forma, estes estudos poderão gerar novos questionamentos e conhecimentos, contribuindo para o avanço da enfermagem brasileira.

Napoleão ${ }^{(2)} \quad$ considera importante 0 desenvolvimento de pesquisas sobre a NIC, pois o melhor conhecimento da mesma pode oferecer bases científicas mais consistentes para o cuidado ao paciente. Estudos realizados ${ }^{(2-4)}$ identificaram diagnósticos de enfermagem (DE) em pacientes pediátricos e adultos submetidos a cirurgias abdominais e internados em unidades de terapia intensiva. A partir dos DE mais frequentes, foram elencadas as intervenções de enfermagem mais executadas. Os autores sugerem novas relações entre diagnósticos e intervenções ainda não descritas na NIC.

Estudo desenvolvido com enfermeiras de São Paulo identificou, dentre as atividades contidas na intervenção denominada controle de líquidos, a importância da realização dessas atividades no cuidado de pacientes cardiopatas com o diagnóstico de enfermagem de excesso de volume de líquidos. Constatou-se que $100 \%$ das atividades eram realizadas muito frequentemente pelos enfermeiros, sendo essenciais para a resolução do problema ${ }^{(5)}$.

Em outro estudo realizado no Brasil, as ações de enfermagem para a Classificação Internacional da Prática de Enfermagem em Saúde Coletiva - CIPESC foram mapeadas às intervenções da $\mathrm{NIC}^{(6)}$. Concluiuse que seria possível que os enfermeiros brasileiros identificassem e utilizassem as intervenções da NIC na prática clínica desses profissionais, caso as mesmas lhes fossem apresentadas.

Considera-se importante determinar o reconhecimento das intervenções da NIC pelos enfermeiros brasileiros nas diversas especialidades clínicas, pois estudos deste tipo auxiliarão o desenvolvimento de sistemas de informação a serem implantados em diversas instituições públicas e privadas do país. É essencial verificar a aplicabilidade das intervenções da NIC à realidade de cuidado de enfermagem no Brasil. Assim, a avaliação de efetividade e qualidade do cuidado de saúde prestado pela enfermagem será facilitada e melhorada ao utilizar linguagem padronizada para designar as intervenções de realizadas.

Este estudo teve por objetivo identificar o uso das intervenções de enfermagem da NIC no Brasil, através do levantamento da frequência de utilização das 486 intervenções descritas na NIC nas diversas especialidades clínicas dos enfermeiros brasileiros.

\section{MATERI AL E MÉTODOS}

Trata-se de estudo descritivo que contou com uma amostra de 222 enfermeiros de todas as regiões do país. A escolha dos participantes do estudo se deu através da busca de endereços eletrônicos de especialistas, mestres e doutores no currículo lattes, banco de dados de participantes em eventos científicos, publicações em revistas, e busca com profissionais de referência em pesquisas na área, totalizando uma população de 2122 enfermeiros. Os mesmos foram contactados via correio eletrônico. O tamanho da amostra foi calculado em um programa estatístico utilizando-se um nível de confiança de 
95\% e erro aceitável de 3\%. Para a coleta de dados foi utilizado um instrumento tipo questionário, elaborado, testado e aplicado pelo grupo de pesquisadores do College of Nursing, University of Iowa, EUA. Utilizou-se a versão traduzida para o português da NIC, terceira edição, contendo 486 intervenções de enfermagem. O questionário foi adaptado à forma eletrônica e enviado pela web, através de correio eletrônico. Cada participante recebeu em seu e-mail um código de usuário e uma senha de acesso ao questionário. Foi esclarecido aos mesmos que, o acesso ao questionário seria entendido como um termo de consentimento em participar da pesquisa.

A coleta de dados foi realizada no período de março a julho de 2006. Durante o período foram enviados e-mails aos participantes com esclarecimentos de dúvidas acerca do preenchimento do instrumento. A coleta eletrônica dos dados possibilitou o acompanhamento passo a passo do número de acessos ao questionário, e do número de respondentes e de não respondentes, assim como estimativas de dados parciais do estudo durante seu desenvolvimento. Os dados retornavam automaticamente para alimentação do banco de dados, especialmente elaborado para o tratamento dos mesmos. Para a análise dos dados utilizou-se o pacote estatístico SPSS, versão 10.0.

Os respondentes determinaram a frequência de utilização das intervenções da NIC na prática clínica. Cada intervenção possuía na frente uma escala onde o enfermeiro discriminava a frequência de sua utilização na prática clínica como: nunca, raramente, mensalmente, semanalmente ou diariamente. Identificaram-se as intervenções centrais utilizadas em cada especialidade clínica segundo a percepção dos enfermeiros. Foram consideradas intervenções centrais aquelas que descrevem a natureza da especialidade $^{(7)}$, e executadas diariamente e/ou semanalmente por mais de $70 \%$ de enfermeiros de cada clínica, conforme a literatura da área. Este grau de concordância é considerado aceitável ${ }^{(8)}$.

Este estudo cumpriu com a Resolução 196/96 sobre pesquisa envolvendo seres humanos e foi aprovado pelo Comitê de Ética em Pesquisa da Universidade Federal de Minas Gerais (Parecer Etic COEP/UFMG no 201/2005).

\section{RESULTADOS E DISCUSSÃO}

Retornaram 222 questionários respondidos, que correspondiam a $10,5 \%$ do total de enfermeiros contactados. Os enfermeiros são assistenciais, trabalham no ensino e na pesquisa. Identificou-se 20 especialidades para os enfermeiros no Brasil distribuídas nas áreas clínica, administrativa e de ensino. Criou-se uma categoria de enfermeiros que não preencheram o campo de especialidade, denominada "não responderam". Verificou-se a participação de enfermeiros das cinco regiões do país, sendo a maioria da região Sudeste $(61,3 \%)$.

Foi identificado o quantitativo das intervenções NIC consideradas centrais em cada especialidade, conforme ilustra a Tabela 1 . 
Tabela 1: Distribuição do quantitativo das intervenções centrais por especialidade segundo a percepção dos enfermeiros brasileiros. Brasil, 2006.

\begin{tabular}{cc}
\hline Especialidade dos enfermeiros & No de intervenções \\
\hline Home Care & $250-51,4 \%$ \\
Cuidados intensivos & $122-25,1 \%$ \\
Saúde mental e psiquiatria & $127-26,1 \%$ \\
Ortopedia & $124-25,5 \%$ \\
Gerontologia & $115-23,6 \%$ \\
Cardiologia & $112-23,0 \%$ \\
Urgência/Emergência & $106-21,8 \%$ \\
Oncologia & $100-20,5 \%$ \\
Neonatologia & $87-17,9 \%$ \\
Práticas alternativas & $83-17,0 \%$ \\
Não responderam & $77-15,8 \%$ \\
Clínica médico-cirúrgica & $62-12,7 \%$ \\
Educação & $57-11,7 \%$ \\
Pediatria & $36-7,4 \%$ \\
Ginecologia & $28-5,7 \%$ \\
Cuidado ambulatorial & $23-4,7 \%$ \\
Controle de infecção e Epidemiologia & $18-3,7 \%$
\end{tabular}

$\mathrm{Na}$ especialidade de cuidado domiciliar foram identificadas como centrais $250(51.4 \%)$ das 486 intervenções da NIC. Pode-se inferir que isto ocorre devido à maioria das intervenções da NIC serem aplicáveis à prática clinica onde o cuidado domiciliar se enquadra. Sabe-se ainda que esta especialidade abrange uma grande variedade de cuidados de enfermagem a diversos tipos de pacientes, e é uma área em expansão da atuação da enfermagem brasileira. Tal fato pode justificar terem sido os enfermeiros desta especialidade os que identificaram um maior número de intervenções da NIC como centrais.

$\mathrm{Na}$ área de administração e gerência somente 2 $(0.4 \%)$ das intervenções foram consideradas como centrais: educação para a saúde e preceptor: estudante. Questiona-se que no Brasil atividades de administração da assistência de enfermagem e que envolvem muitas vezes cuidados indiretos aos pacientes como conferência de carrinho de ressuscitação, controle do ambiente e segurança do trabalhador, avaliação crítica realizada por colegas, delegação, gerenciamento de recursos financeiros e outras não foram identificadas como centrais pelos enfermeiros brasileiros.

Destaca-se que os enfermeiros de saúde pública consideraram um pequeno número de intervenções (3-0,6\%) como centrais à sua prática clínica, são elas: ouvir ativamente, educação para saúde e avaliação da saúde. Em inventário vocabular realizado durante $o$ projeto de pesquisa da Classificação Internacional da Prática de Enfermagem em Saúde Coletiva (CIPESC) no Brasil 3.429 ações de enfermagem foram identificadas ${ }^{(9)}$. Estudo realizado nos Estados Unidos ${ }^{(10)}$ acerca do uso de diagnósticos e intervenções de enfermagem na prática em saúde pública identificou 106 intervenções da NIC, sendo que nenhuma das dez intervenções mais frequentes coincidem com aquelas identificadas pelos enfermeiros brasileiros.

Estudo realizado para mapear as 105 ações incluídas em um dos instrumentos do projeto de Classificação Internacional da Prática de Enfermagem em Saúde Coletiva (CIPESC) realizado no Brasil às intervenções de enfermagem da NIC foi conduzido e uma listagem de 110 intervenções NIC foram mapeadas àquelas ações do instrumento. Considerou-se ainda que os termos utilizados no CIPESC puderam ser mapeados às intervenções NIC e que a NIC poderia ser usada para descrever tanto a prática de enfermagem em saúde coletiva como hospitalar ${ }^{(11)}$.

Das 486 intervenções descritas na NIC, 378 $(77,7 \%)$ foram identificadas como essenciais pelo conjunto de enfermeiros das diversas especialidades. Estas intervenções foram distribuídas segundo os domínios da NIC. O domínio fisiológico complexo apresentou o maior número de intervenções (104 $27,5 \%)$, entre aquelas consideradas centrais, seguido do domínio comportamento (89 - 23,5\%). No domínio fisiológico complexo estão agrupadas as intervenções relacionadas aos cuidados de suporte à regulação homeostática. O domínio comportamento descreve as intervenções referentes aos cuidados de 
suporte ao funcionamento psicossocial e que facilitam mudanças no estilo de vida(1). As enfermeiras de unidades de clínicas médicas e cirúrgicas, tanto na Coréia como nos Estados Unidos, também consideraram intervenções de enfermagem do domínio fisiológico complexo como as mais empregadas nas suas especialidades ${ }^{(12)}$

As intervenções NIC consideradas centrais em mais de onze especialidades clínicas (mais de $50 \%$ do total de especialidades) estão apresentadas na Tabela 2.

Tabela 2: Distribuição das intervenções NIC identificadas como centrais por enfermeiros de mais de 11 especialidades clínicas. Brasil, 2006.

\begin{tabular}{|c|c|}
\hline I ntervenções Centrais & Frequência (n especialidades) \\
\hline Avaliação de saúde & 17 \\
\hline Educação para saúde & 16 \\
\hline Documentação & 16 \\
\hline Ouvir atentamente & 15 \\
\hline Presença & 15 \\
\hline Controle de infecção & 15 \\
\hline Cuidado com Sondas e drenos: bexiga & 14 \\
\hline Administração de medicamentos: Oral & 14 \\
\hline Preceptor: estudante & 14 \\
\hline Monitorização de sinais vitais & 14 \\
\hline Apoio médico & 14 \\
\hline Administração de analgésico & 13 \\
\hline Administração de medicamentos & 13 \\
\hline Inserção endovenosa (EV ou IV) & 13 \\
\hline Terapia Endovenosa & 13 \\
\hline Administração de medicamentos: endovenosa & 12 \\
\hline Controle do ambiente: conforto & 12 \\
\hline Cuidados com lesões & 12 \\
\hline Controle de medicamentos & 12 \\
\hline Monitorização de eletrólitos & 12 \\
\hline Assistência ao auto cuidado & 11 \\
\hline Assistência ao auto cuidado: banho higiene & 11 \\
\hline Cuidado com repouso no leito & 11 \\
\hline Passagem de plantão & 11 \\
\hline Supervisão & 11 \\
\hline Suporte Emocional & 11 \\
\hline
\end{tabular}

Pode-se afirmar que as intervenções identificadas como centrais refletem a prática da enfermagem brasileira. Tais intervenções envolvem cuidados diretos e indiretos ao paciente. Vale destacar que, cuidados básicos como ouvir ativamente e presença são realizados no cotidiano da enfermagem e nem sempre são valorizados ou identificados como tal. Estas foram identificadas como centrais em 15 (71,4\%) das 20 especialidades e também pela categoria de enfermeiros que não especificou sua especialidade.

Enfermeiras coreanas selecionaram 202 intervenções NIC, sendo 09 utilizadas por mais de $50 \%$ delas $^{(12)}$. Por sua vez, enfermeiras americanas identificaram 68 intervenções como sendo usadas por mais de $43 \%$ delas $^{(12)}$.

A partir dos resultados do presente estudo identificou-se que tanto as enfermeiras brasileiras, como as coreanas e americanas julgaram 12 intervenções NIC como centrais à sua prática (documentação, cuidados com sondas e drenos: bexiga, administração de medicamentos: oral, monitorização de sinais vitais, administração de medicamentos, inserção endovenosa, administração endovenosa, terapia endovenosa, cuidado com lesões, controle de medicamentos, cuidados com repouso no leito e suporte emocional ${ }^{(12)}$.

Algumas intervenções foram semelhantes às das enfermeiras coreanas, a saber: ouvir atentamente, educação para a saúde e passagem de plantão. Foram comuns com as enfermeiras americanas: controle de eletrólitos, controle de infecção e assistência ao autocuidado ${ }^{(12)}$.

Observa-se que estudos já têm sido conduzidos para identificar na prática intervenções de enfermagem NIC em grupos de pacientes, como o realizado em unidades de um hospital escola no Brasil, a partir da análise de pacientes idosos. Encontrou-se quatro diagnósticos de enfermagem prevalentes entre $62^{(13)}$. Destes, cuidados principais 
que poderiam ser mapeados à NIC foram identificados. Entre eles, terapia endovenosa foi o único cuidado referente a uma intervenção NIC comum àquelas identificadas como centrais no presente estudo.

Estudos têm sido realizados utilizando a NIC, tanto no âmbito nacional como internacional. Estudo para identificar a produção sobre a NIC foi conduzido, identificando-se que tratar-se da aplicação da NIC na prática, da comparação de linguagens em sistemas informatizados e do seu uso na assistência, pesquisa e ensino. Os estudos também têm apresentado a classificação e sido propostos para contribuir na construção e desenvolvimento da taxonomia, além de validação de intervenções para grupos selecionados de pacientes $^{(14)}$. Assim, compreende-se que o presente trabalho é mais uma contribuição ao desenvolvimento da NIC. Esta tem sido considerada um recurso importante para melhorar a prática da enfermagem no ensino, pesquisa e assistência.

\section{CONCLUSÕES}

A utilização do método eletrônico para a coleta de dados mostrou-se vantajosa quando comparada aos métodos tradicionais, tendo em vista que reduziu os custos com a pesquisa e proporcionou que um número significativo de enfermeiros tivesse acesso ao questionário via web, podendo participar da pesquisa.

Os resultados mostraram que as intervenções NIC são aplicáveis à prática clínica da enfermagem brasileira, uma vez que $77,7 \%$ das 486 intervenções descritas foram consideradas centrais pelos enfermeiros brasileiros. As intervenções identificadas como centrais nas diversas especialidades descrevem a natureza das mesmas. O uso das intervenções NIC na prática clínica pode ajudar enfermeiros a comunicar o cuidado que executam.

A utilização das intervenções NIC na construção de bancos de dados e sistemas de informação em enfermagem facilitará a prática da enfermagem e fornecerá dados para o desenvolvimento de pesquisas que contribuirão para dar uma maior visibilidade do trabalho executado por esses profissionais.

No Brasil está ocorrendo um aumento expressivo na utilização de sistemas informatizados, em especial na rede hospitalar. Os enfermeiros precisam se aproximar de tecnologias inovadoras, conhecer e utilizar as etapas do método científico na prática de enfermagem e cada vez mais tentar empregar linguagens uniformizadas para designar diagnósticos, intervenções e resultados de enfermagem que irão favorecer a construção das bases de dados dos referidos sistemas.

\section{REFERÊNCI AS}

1. Mccloskey JJ, Bulecheck G. Classificação de Intervenções de Enfermagem. Porto Alegre: Artemed; 2004.

2. Napoleão AA. Estudo da aplicabilidade de intervenções da NIC no atendimento a crianças com o diagnóstico de enfermagem "desobstrução ineficaz de vias aéreas relacionada à presença de via aérea artificial" em um centro de terapia intensivo pediátrico [thesis]. São Paulo: Escola de Enfermagem de Ribeirão Preto/USP; 2005. 301p.

3. Yom YH, Chi SA, Yoo HS. Application of nursing diagnoses, interventions, and outcomes to patients undergoing abdominal surgery in Korea. Int J Nurs Terminol Classif. 2002;13(3):77-87.

4. Lucena AF. Mapeamento dos diagnósticos e intervenções de enfermagem em uma unidade de terapia intensiva [thesis]. São Paulo: Escola Paulista de Medicina/UNIFESP; 2006. 193p.

5. Guimarães HCCP, Barros ABL. Controlar líquidos: uma intervenção de enfermagem para o paciente com excesso de volume de líquidos. Rev Latino-am Enfermagem. 2003; 11(6): 734-41.

6. Chianca TCM. Mapeamento das ações de enfermagem do CIPESC às intervenções de enfermagem da NIC. Rev Bras Enferm. 2003; 56(5): 513-8.

7. Mccloskey J, Bulechek G, Donahue W. Nursing Interventions Core to Specialty Practice. Nurs Outlook; 1998;46(2): 67-76.

8. Batista CG. Concordância e fidedignidade na observação. Psicologia. 1977;3(2): 39-49.

9. Garcia TR, Nóbrega MML. Projeto CIPESC/ABEn/CIE: I nventário vocabular de fenômeos e ações de enfermagem em saúde coletiva. In: Garcia TR, Nobrega MML. Sistemas de classificação da prática de enfermagem: um trabalho coletivo. J oão Pessoa: ABEn/Idéia; 2000. p. 83-170.

10. Rivera JC, Parris KM. Use of nursing diagnoses and interventions in public health nursing pratice. Int J Nurs Terminol Classif. 2002; 13(1): 15- 23.

11. Chianca TCM. Mapeamento das ações de enfermagem do CIPESC às intervenções da NIC. Rev Bras Enferm. 2003; 56(5): 513-18.

12. Lee $E$, Lee $M$. Comparison of nursing interventions performed by medical-surgical nurses in Korea and United States. Int J Nurs Terminol Classif. 2006; 17(2): 108- 17.

13. Almeida MA, Aliti GB, Franzen E, Thomé EGR, Unikovsky MR, Rabelo $E R$, et al. Diagnósticos de enfermagem e intervenções prevalentes no cuidado ao idoso hospitalizado. Rev. Latino-am Enfermagem. [Internet]. 2008 [cited 2009 aug 15]; 16(4):707-11. Available http://www. scielo.br/pdf/rlae/v16n4/pt 09.pdf. 14. Napoleão AA, Chianca TCM, Carvalho EC, Dalri MCB. Análise da produção científica sobre a classificação das intervenções de enfermagem (NIC) 
de 1980 a 2004. Rev. Latino-am Enfermagem.

[Internet]. 2006 [cited 2009 aug 15];14(4):608-13.

Available

from:

http://www.scielo.br/pdf/rlae/v14n4/v14n4a20.pdf.

Artigo recebido em 09.09.08.

Aprovado para publicação em 03.06.09.

Artigo publicado em 30.09.09. 